\title{
Acupuncture and stroke rehabilitation
}

\author{
Hongmei Wu MD PhD
}

Previously published at www.cmaj.ca

$\infty \infty$

See related research article by Kong and colleagues, page 1723

$\mathrm{A}$ cupuncture has been used in traditional Chinese medicine for more than 3000 years as a treatment for many diseases and is especially well accepted in China for rehabilitation after stroke. ${ }^{1}$ Several systematic reviews have suggested that acupuncture is effective in stroke rehabilitation; ${ }^{2-}$ ${ }^{6}$ however, the results were inconclusive because of extensive heterogeneity across trial outcomes and a high risk of bias.

In this issue, Kong and colleagues report their finding of no specific effect of acupuncture on functional recovery after stroke. ${ }^{7}$ For their systematic review and meta-analysis, they included sham-controlled randomized clinical trials identified through a search of 25 databases and 12 major Korean traditional medicine journals from their inception to October 2009. Trials that compared acupuncture (with or without electrical stimulation) and sham acupuncture regardless of the age and sex of patients, and the stages (acute, subacute and chronic) and types of stroke (ischemic and hemorrhagic) were included. The outcome measures evaluated were neurologic deficit, activities of daily living, motor recovery, quality of life and adverse events. The methodologic quality of the trials was assessed using the Cochrane risk-of-bias criteria and the PEDro (Physiotherapy Evidence Database) scale. Kong and colleagues identified 664 potentially relevant studies, 10 of which were included in their systematic review.

Lack of blinding in randomized trials has been shown to be associated with more exaggerated odds ratios for intervention effect, by $9 \%$ on average. ${ }^{8}$ Previous systematic reviews included not only randomized clinical trials that compared acupuncture with placebo or sham acupuncture but also ones that compared acupuncture with no acupuncture or other treatment. ${ }^{2-6}$ Kong and colleagues included only randomized clinical trials that compared acupuncture with sham acupuncture. Sham acupuncture includes the use of needles on nonacupuncture points or electrostimulation via electrodes attached to the skin. With the use of sham acupuncture as a control, participants are expected to be blinded to treatment allocation, with the result being a reduction in the risk of performance bias. Therefore, the results of the effects of acupuncture reported in sham- or placebo-controlled trials should be more valid and reliable. A recent systematic review and meta-analysis showed that the use of sham acupuncture may contribute to study heterogeneity and affect the results

\section{Key points}

- Although previous systematic reviews have suggested that acupuncture is effective in stroke rehabilitation, a new systematic review and meta-analysis does not show a positive effect.

- Most of the reviews are based on small, heterogeneous trials.

- Sham acupuncture may not be inert.

- The question of acupuncture's effectiveness in stroke rehabilitation can be answered only with rigorously designed, large, multicentre randomized trials.

(odds ratio $0.57,95 \%$ confidence interval $0.30-0.41) .{ }^{6}$ It also showed no difference in effectiveness between sham acupuncture and true acupuncture. ${ }^{6}$

The lack of a positive effect of acupuncture in stroke rehabilitation reported by Kong and colleagues should be interpreted with caution because of the following limitations.

First, the studies included in their systematic review were small (8-120 participants). Although one of the benefits of meta-analysis is that a synthesis of inadequately powered studies can yield interesting findings, a large number of studies would be required in order to show conclusive findings. Researchers should ensure that their efficacy trials are powered appropriately. An inadequately powered meta-analysis may have been one of the reasons for the lack of effectiveness of acupuncture on stroke rehabilitation.

Second, the quality of acupuncture treatment is closely related to its effectiveness. Kong and colleagues report that the degree of confidence they had in the quality of the acupuncture varied from $55 \%$ to $93 \%$ (mean $82.4 \%$ ).

Third, in sham-controlled trials, there may be concern about whether concealment of treatment allocation was successful. Some potential factors that influence the applicability of sham acupuncture include not only interrater variability, but also the patient's knowledge and experience of acupuncture, the visual impact of needling and other contextual effects. ${ }^{9}$ For patients who have had acupuncture previously,

Hongmei Wu is with the Department of Geriatrics, West China Hospital, Sichuan University, Chengdu, Sichuan, China.

CMAJ 2010. DOI:10.1503/cmaj.101311

All editorial matter in CMAJ represents the opinions of the authors and not necessarily those of the Canadian Medical Association. 
sham acupuncture may produce familiar sensations of needling. As a result, sham acupuncture may not be inert. Thus, no differences in effectiveness between study groups would emerge from the review by Kong and colleagues.

In the face of these limitations, it is premature to refute the effects of acupuncture in the treatment of stroke rehabilitation. Rigorously designed, large, multicentre randomized trials are needed to assess the effects of acupuncture on stroke rehabilitation further.

This commentary was solicited and has not been peer reviewed.

Competing interests: None declared.

\section{REFERENCES}

1. Rabinstein AA, Shulman LM. Acupuncture in clinical neurology. Neurologist 2003;9:137-48

2. Park J, Hopwood V, White AR, et al. Effectiveness of acupuncture for stroke: a systematic review. J Neurol 2001;248:558-63.
3. Sze FK, Wong E, Or KK, et al. Does acupuncture improve motor recovery after stroke? A meta-analysis of randomized controlled trials. Stroke 2002;33: 2604-19.

4. Wu HM, Tang JL, Lin XP, et al. Acupuncture for stroke rehabilitation [review] Cochrane Database Syst Rev 2006;(3):CD004131.

5. Zhang SH, Liu M, Asplund K, et al. Acupuncture for acute stroke [review]. Cochrane Database Syst Rev 2005;(2):CD003317.

6. Wu P, Mills E, Moher D, et al. Acupuncture in poststroke rehabilitation: a systematic review and meta-analysis of randomized trials. Stroke 2010;41:e171-9.

7. Kong JC, Lee MS, Shin BC, et al. Acupuncture for functional recovery after stroke: a systematic review of sham-controlled randomized clinical trials. CMAJ 2010;182:1723-9.

8. Pildal J, Hróbjartsson A, Jørgensen KJ, et al. Impact of allocation concealment on conclusions drawn from meta-analyses of randomized trials. Int J Epidemiol 2007; 36:847-57.

9. Tsukayama H, Yamashita H, Kimura T, et al. Factors that influence the applicability of sham needle in acupuncture trials: two randomized, single-blind, crossover trials with acupuncture-experienced subjects. Clin J Pain 2006;22:346-9.

Correspondence to: Dr. Hongmei Wu, Department of Geriatrics, West China Hospital, Sichuan University, No. 37, Guo Xue Xiang, Chengdu, Sichuan 610041, China; drwhm@163.com

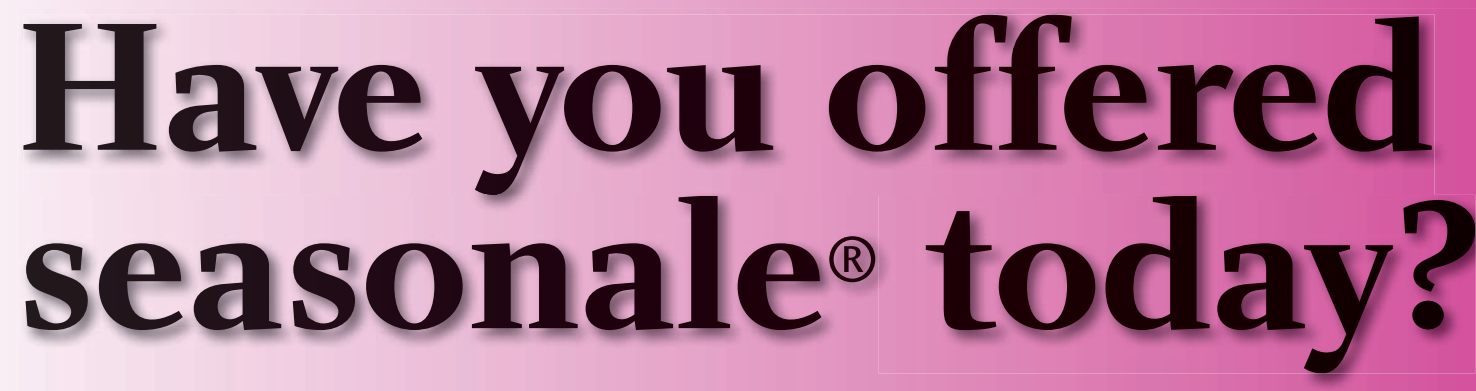

Seasonale is indicated for the Prevention of Pregnancy.

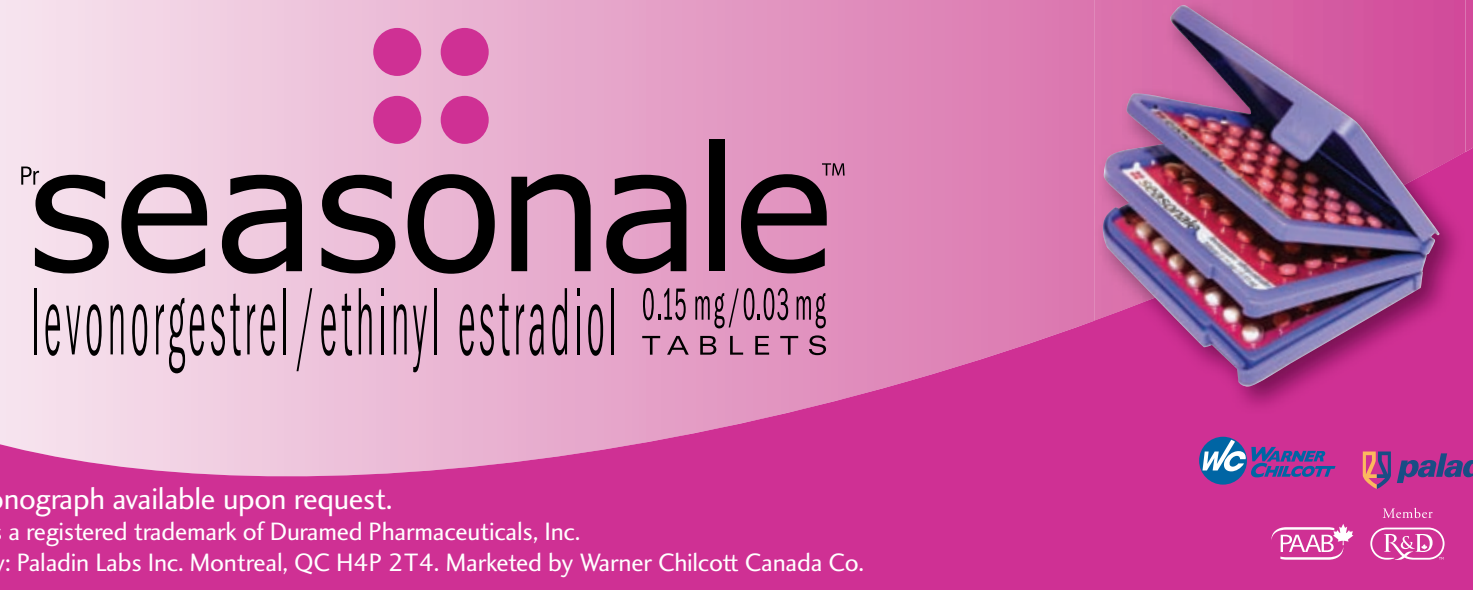

